\title{
ANTIMICROBIAL ACTIVITY OF AH PLUS AND CEASEAL SEALERS WITH AND WITHOUT THE ADDITION OF SILVER NANOPARTICLES AGAINST ENTEROCOCCUS FAECALIS
}

\author{
Nehal Nabil Roshdy ${ }^{*}$, Adel AbdelWahed ${ }^{* *}$ and Hisham Elshishtawy ${ }^{* * *}$
}

\begin{abstract}
Aim: The aim of the present study was to investigate the antimicrobial effect of AH Plus and Ceraseal sealers with and without the incorporation of silver nanoparticles against Enterococcus faecalis using direct contact assay.

Methodology: Silver nanoparticles were prepared to be used in a gel form using the chemical reduction method. Antibacterial activity was assessed using the direct contact test (DCT) against Enterococcus faecalis ATCC 35550). Fifteen tubes were set for each sealer in duplicate as follow: Group (I): Ceraseal (Meta Biomed Co., Cheongju, Korea), Group (II): Ceraseal\& Nano-silver gel, Group (III): AH Plus (Dentsply/Maillefer, Konstanz, Germany) and Group (IV): AH Plus \& Nanosilver gel. Colony counts were detected for all groups at different time intervals $(1,24,168 \mathrm{~h})$.

Results: DCT results revealed that AH Plus had higher antibacterial activity against Enterococcus faecalis at different time intervals than Ceraseal sealer unaffected by silver nanoparticles gelincorporation. But the incorporation of silver nanoparticles gel significantly enhanced their antibacterial effect. All sealers had lost much of their antibacterial effects at 7-day intervals.
\end{abstract}

Conclusion: AH Plus sealer had higher antibacterial activity against Enterococcus faecalis than CeraSeal sealer. Adding Silver nanoparticles gel to both sealers improved their antibacterial activity.

KEYWORDS: AH Plus sealer, CeraSeal sealer, Silver nanoparticles, Direct contact test, Enterococcus faecalis.

* Associate Professor, Endodontic Department, Cairo University

** Endodontic Department- Faculty of Oral And Dental Medicine- Future University

*** Associate Professor, Department of Microbial Genetics, Agricultural Genetic Engineering Research Institute, Agriculture Research Center, Cairo, Egypt. 


\section{INTRODUCTION}

Post-endodontic treatment failures are frequently increasing and present a high risk of complicated infections in patients. Research stated that up to $10 \%$ of root canal treatments fail because of residing bacteria in the root canal ${ }^{1}$. Enterococcus faecalis represents the most resistant species responsible for failed root canal treatment. Thus, elimination of bacteria from the root canal is crucial to ensure the success of the root canal treatment ${ }^{2}$.

Multiple improvements have been achieved in the protocols of endodontic chemo-mechanical disinfection used today, yet they are still not able to provide $100 \%$ sterility of the root canal complex. Thereby, the antimicrobial activity of root canal sealer aid in the elimination of the residual bacteria left in the root canal system unaffected by various chemo-mechanical preparation protocols ${ }^{3}$. This leads to continuous improvement in the products of endodontic sealers to exhibit a high antimicrobial activity, reduce or inhibit the growth of microorganisms and enhance the repair process of periapical tissues.

Lately, nanotechnology has been utilized in creation of novel bio-materials. Silver nanoparticles have received considerable attention because of their good biocompatibility with human cells ${ }^{4}$, low bacterial resistance, broad spectrum antibacterial nature due to sustained ion release, and inhibiting bacterial growth at lower concentrations than antibiotics ${ }^{5}$.

Variety of sealers are currently available; epoxyresin based sealers are considered the most widely used due to their biological, physicochemical and sealing abilityproperties ${ }^{6}$, together with the probability of chemical bonding to dentin collagen ${ }^{7}$.

Recently, bioceramic-based sealers (calcium silicate and /or calcium phosphate) became favorable because of their biological and physical properties; i.e. alkaline $\mathrm{pH}$, lack of shrinkage and chemical stability ${ }^{8}$. Above all, bioceramic sealers shows enhanced setting properties due to deposition of crystalline structure similar to that of the tooth and bone apatite materials that improves the sealer to root dentin bonding ${ }^{9}$.

Thus, the aim of the present study was to investigate the antimicrobial effect of $\mathrm{AH}$ Plus (Dentsply/Maillefer, Konstanz, Germany) and Ceraseal sealer (Meta Biomed Co., Cheongju, Korea) with and without the incorporation of silver nanoparticles against Enterococcus faecalis using the direct contact assay. The null hypothesis was that there would be no significant difference among the test groups.

\section{METHODOLOGY}

\section{Silver Nanoparticles(SNP) Preparation and characterization}

Silver nanoparticles were prepared by the chemical reduction method as reported by Turkevich 10 and Lee and Meisel 11. A solution of $\mathrm{AgNO}_{3}$ had been utilized as Ag1+ ions precursor. PVP was used as a stabilizing agent and borohydrateacted as a mild reducing agent. When the color of the solution turned slowly into grayish yellow, this indicated the reduction of the $\mathrm{Ag} 1+$ ion to $\mathrm{Ag}$ nanoparticles.

\section{SNP Gel}

$0.4 \mathrm{gm}$ of Carboxymethyl cellulose (Loba CHIME, india) was sprinkled gently and gradually over the solution of Silver nanoparticles 200ppm under mild temperature with vigorous stirring to get homogenous gel. The gel was mixed with sealer in ratio $1: 1$ to get final sealer of $100 \mathrm{ppm}$ Silver nanoparticles with an average size of less than 20nm and Spherical shape under a transmission electron microscope.

\section{Antibacterial test}

Direct Contact Test (DCT) was conducted by placing 50mg of freshly mixed sealer in sterile flat- 
bottom, screw-capped test tubes. Fifteen tubes were used for each group in duplicate as follow:

- Group (I): Ceraseal (Meta Biomed Co., Cheongju, Korea)

- Group (II): Ceraseal\& Nano-silver gel

- Group (III): AH Plus (Dentsply/Maillefer, Konstanz, Germany)

- Group (IV): AH Plus \& Nano-silver gel

After that, $50 \mu \mathrm{L}$ of $0.5 \mathrm{ml}$ McFarland standard suspension $\left(1.5 \times 10^{6} \mathrm{CFU} / \mathrm{ml}\right)$ of Enterococcus faecalis ATCC 35550 was pipetted and applied over each sealer. Tubes were incubated at $37^{\circ} \mathrm{C}$ to confirm direct contact between the bacteria and the sealers. The tubes were divided into three equal sub-groups according to the tested time interval. E. faecalis suspension was allowed to be in contact with the tested sealer for 1, 24, and $168 \mathrm{~h}$.

\section{Colony Count:}

The bacterial suspension was diluted by adding $600 \mu \mathrm{L}$ of sterile nutrient broth to the screw-capped tubes. Then, $50 \mu \mathrm{L}$ of suspension was drawn from each vial and spread over Mac Conkey agar to detect the colony count manually. Colony counts of all the tested groups at different time intervals $(1,24$ and $168 \mathrm{~h}$ ) were conducted in the same manner to detect the immediate and delayed antimicrobial efficacy against E. faecalis. A suspension of E.faecalis ATCC 35550 in normal saline was taken as the positive control, sub cultured and colony count was detected at 1, 24, $168 \mathrm{~h}$ interval. Another set of test tube containing tested material without bacterial inoculates served as negative control.

\section{Statistical analysis}

Numerical data were presented as mean and standard deviation (SD) values. Data were explored for normality by checking the data distribution and using Kolmogorov-Smirnov and ShapiroWilk tests, it showed a parametric distribution.
One-ANOVA followed by Tukey's post hoc test was used for intergroup comparisons, while oneway repeated measures ANOVA followed by a comparison of main effects utilizing Bonferroni correction was used for intragroup comparisons. The significance level was set at $\mathrm{P} \leq 0.05$ within all tests. Statistical analysis was performed with IBM ${ }^{\circledR}$ SPSS ${ }^{\circledR}$ Statistics Version 26 for Windows.

\section{RESULTS}

Mean and standard deviation (SD) values for bacterial count in different groups were presented in Table (1) and Figure (1)

\section{Intergroup comparison:}

1- Intergroup comparison for all time intervals showed significant difference between

2- Different groups $(\mathrm{p}<0.001)$. Control group showed the highest value of bacterial count,

3- Followed by Ceraseal group, Ceraseal\&Nano group, AH Plus group and AH Plus \& Nano

4- Group while negative control group had the lowest mean value. Pairwise comparisons showed

5- Different groups to be significantly different from each other except AH plus \& Nano group

6- and Negative control group $(\mathrm{p}<0.001)$

For all the time intervals $(1,24,168 \mathrm{~h})$, there was significant difference among different groups $(p<0.001)$. The control group showed the highest value of bacterial count, followed by Group (I): Ceraseal group, Group (II): Ceraseal \& Nanosilver gel group, Group (III): AH Plus group and Group (IV): AH Plus \& Nano-silver gel while the negative control group had the lowest mean value. Pairwise comparisons showed different groups to be significantly different from each other except for Group (IV): AH plus \& Nano group and the negative control group $(\mathrm{p}<0.001)$. 
TABLE (1): Mean and standard deviation (SD) values for bacterial count (CFU/mL)in different groups

\begin{tabular}{|c|c|c|c|c|c|c|c|}
\hline \multirow[b]{2}{*}{ Time } & \multicolumn{6}{|c|}{$(\mathrm{CFU} / \mathrm{mL})(\mathrm{Mean} \pm \mathrm{SD})$} & \multirow[b]{2}{*}{ p-value } \\
\hline & $\begin{array}{c}\text { Negative } \\
\text { control }\end{array}$ & Control & Ceraseal & $\begin{array}{c}\text { Ceraseal } \\
\& \text { Nano }\end{array}$ & AH Plus & $\begin{array}{c}\text { AH Plus \& } \\
\text { Nano }\end{array}$ & \\
\hline $1 \mathrm{~h}$ & $0.00 \pm 0.00^{\mathrm{E}}$ & $342.00 \pm 23.87^{\mathrm{Ab}}$ & $216.20 \pm 9.86^{\mathrm{Bb}}$ & $163.20 \pm 12.56^{\mathrm{Cb}}$ & $39.60 \pm 6.27^{\mathrm{Db}}$ & $1.20 \pm 1.30^{\mathrm{Eb}}$ & $<0.001 *$ \\
\hline $24 \mathrm{~h}$ & $0.00 \pm 0.00^{\mathrm{E}}$ & $473.40 \pm 14.93^{\mathrm{Aa}}$ & $218.60 \pm 11.26^{\mathrm{Bb}}$ & $160.40 \pm 13.92^{\mathrm{Cb}}$ & $45.60 \pm 4.83^{\mathrm{Db}}$ & $1.60 \pm 1.67^{\mathrm{Eb}}$ & $<0.001 *$ \\
\hline $168 \mathrm{~h}$ & $0.00 \pm 0.00^{\mathrm{E}}$ & $492.00 \pm 10.95^{\mathrm{Aa}}$ & $256.60 \pm 11.24^{\mathrm{Ba}}$ & $180.80 \pm 10.71^{\mathrm{Ca}}$ & $81.40 \pm 9.56^{\mathrm{Da}}$ & $10.80 \pm 4.38^{\mathrm{Ea}}$ & $<0.001 *$ \\
\hline
\end{tabular}

Different upper and lowercase superscript letters indicate a statistically significant difference within the same horizontal row and vertical column respectively*; significant $(p \leq 0.05)$ ns; non-significant $(p>0.05)$

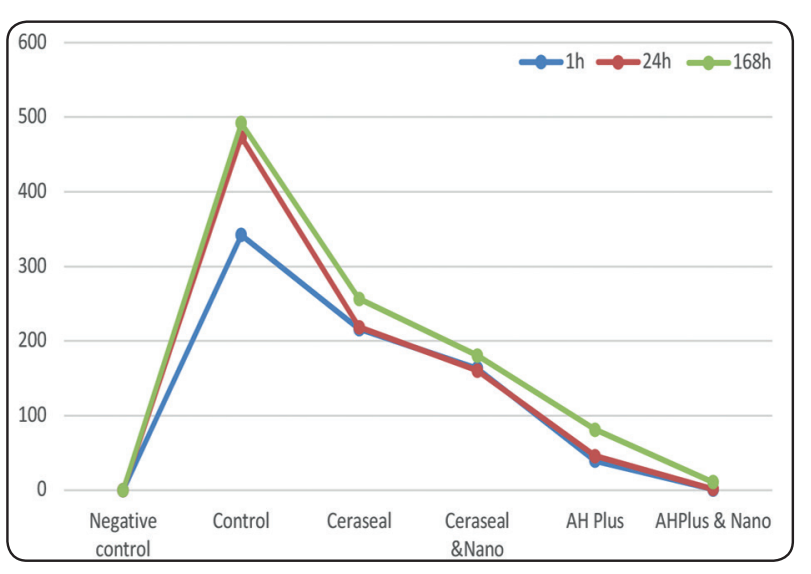

Fig. (1) Line chart showing average bacterial count (CFU/mL) in different groups.

\section{Intragroup comparison}

All the tested groups showed a significant difference between different time intervals $(\mathrm{p}<0.001)$. Pairwise comparisons showed value recorded at168 $\mathrm{h}$ to be significantly higher than values recorded at other intervals $(\mathrm{p}<0.001)$ and there was no significant difference between values recorded in $1 \mathrm{~h}$ and $24 \mathrm{~h}$ ( $\mathrm{p}>0.05)$. For Group (I): Ceraseal, Group (III): AH Plus and Group (IV): AH Plus and Nanosilver gel; The highest value of bacterial count was recorded at $168 \mathrm{~h}(256.60 \pm 11.24),(81.40 \pm 9.56)$ and $(10.80 \pm 4.38)$ respectively followed by $24 \mathrm{~h}$ $(218.60 \pm 11.26),(45.60 \pm 4.83)$ and $(1.60 \pm 1.676)$ respectively, while the lowest value was found at

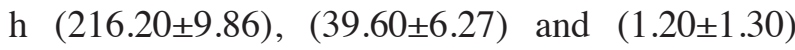
respectively. On the other hand, Group (II):
Ceraseal\& Nano-silver gel. The highest value of bacterial count was found at $168 \mathrm{~h}(180.80 \pm 10.71)$, followed by $1 \mathrm{~h}$ interval $(163.20 \pm 12.56)$ while the low-

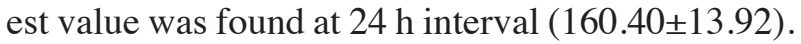

\section{DISCUSSION}

Failure of root canal treatment is usually attributed to the persistence of bacteria especially E.faecalis in the root canal system ${ }^{12}$. Thus, using an endodontic sealer with antibacterial properties is required to eliminate residual microbial infections that have survived the chemo-mechanical instrumentation and irrigation protocols ${ }^{13}$.

E. faecalis (Gram positive, facultative anaerobic microbe) is usually associated with failed root canal treatment cases. It has the ability to survive in the root canal either alone or with other microbes14. $E$. faecalis is difficult to be eradicated from the root canal as it can penetrate the dentinal tubules and adhere to dentinal collagen ${ }^{15}$.

This study aimed to investigate the antimicrobial effect of AH Plus and Ceraseal sealers with and without the incorporation of Silver nanoparticles against E.faecalis using the direct contact assay.

The direct contact test is considered the most accepted antimicrobial test for endodontic sealers 16.The test is considered quantitative and reproducible in mimicking the contact between microorganisms and endodontic sealers inside 
the canal space, providing information about the bactericidal effect ${ }^{17}$, thus providing reliable and relevant results ${ }^{18,19}$. Though, Agar diffusion test (ADT) has been widely used to investigate the antimicrobial activity of sealers, the Editorial Board of the Journal of Endodontics 2007 showed that its results are considered questionable due to agar viscosity, the lack of standardization of inoculum density, plate-storage condition and dependency on the solubility and diffusion characteristic of both the test material and media.

Our results showed that the highest antibacterial effect was reported to Group (IV): AH plus \& Nano silver gel group followed by Group (III): AH Plus group followed by Group (II): Ceraseal\& Nano silver gel group and the least antibacterial activity was related to Group (I): Ceraseal group. Pairwise comparisons showed significant differences among the groups $(\mathrm{p}<0.001)$.

Previous studies demonstrated a superior antibacterial efficiency of AH Plus sealer against E. faecalis when compared to bioceramic sealer (SureSeal) 20 and (Endosequence BC) against $E$. faecalis $^{21,22}$.

AH Plus presents high antibacterial effectiveness due to its release of bisphenol-A-diglycidyl ether during polymerization 23 . While, the antibacterial effect of the bioceramic sealer is related to the combination of high $\mathrm{pH}$, hydrophilicity and active calcium hydroxide diffusion ${ }^{13,22}$.

Our results showed that the addition of silver nano particles to both sealers (AH Plus and CeraSeal) enhanced the antibacterial effect both sealers significantly when compared with conventional sealer $(\mathrm{P}<0.001)$. Incorporation of nanoparticulate drugs to endodontic sealers aims at deeper penetration into the dentinal tubules and into the microbial cells. Adding nanosilver to dental biomaterials and cement shows a positive antimicrobial effect ${ }^{24,25}$. The antimicrobial effect of silver nanoparticles could be attributed to the high surface area to volume ratio and unique physio- chemical properties of silver ions ${ }^{26}$. Moreover, silver ions are positively charged nanoparticles that interact electrostatically with negatively charged bacterial cells causing altered cell permeability 27. Furthermore, silver nano particles interact with multiple targets in the microbial cell; cell membrane, plasmids and enzymes. Thus, it is unlikely for microorganism to develop resistance to silver when compared with antibiotics ${ }^{28}$.Our findings are consistent with Krishnan et al in $2015^{27}$ who reported that silver nanoparticles have potential bactericidal effects on E.faecalis.

All the tested groups showed significant difference between different time intervals $(\mathrm{p}<0.001)$. Pairwise comparisons showed mean values of bacteria recorded at $168 \mathrm{~h}$ to be significantly higher than values recorded at other intervals $(\mathrm{p}<0.001)$. There was no significant difference between values recorded at $1 \mathrm{~h}$ and $24 \mathrm{~h}(\mathrm{p}>0.05)$.

The endodontic sealers have shown to provide the highest antimicrobial effectiveness immediately after spatulation, followed by a gradual loss in the antimicrobial effect over time ${ }^{29}$. The results were consistent with Pizoo et al 30 and Shakya et al ${ }^{31}$, who showed that fresh sealers have antibacterial effects that decrease with time. AH Plus presents good flow, thereby diffusing into the dentinal tubules and creating microbial inhibition by means of entombment ${ }^{32}$. It has been reported that material-released formaldehyde in the polymerization process, resulting in the sealers antibacterial property ${ }^{33}$. On the other hand, Kayaoglu et al. (2005) ${ }^{34}$ reported that fresh AH-Plus sealer had antibacterial activity against $E$. faecalis that diminished at $24 \mathrm{~h}$ and 7-day old samples. This could be attributed to the ease of diffusion of the antibacterial component into the surrounding environment before setting of the material $^{35}$.

In contrast, bioceramic sealers exhibit hydrophilic properties. On contact with dentinal moisture, calcium silicates undergo a hydration reaction which results in the formation of calcium silicate 
hydrogel and calcium hydroxide 36. Calcium hydroxide partially reacts with calcium phosphate forming hydroxyapatite and water. The water formed, in turn, re-initiates the cycle to produce more calcium silicate hydrogel and calcium hydroxide, resulting in an increase in $\mathrm{pH}(>12.5)$. By the time the sealer sets, its $\mathrm{pH}$ also reduces to about 9.14 , consequently lessen its antibacterial efficacy. It had been reported that their antimicrobial properties are greatly diminished 7 days after mixing ${ }^{37}$. Zhang et al. (2009) ${ }^{13}$ reported the antimicrobial effect of bioceramic sealer after $24 \mathrm{~h}$ that greatly decreased 7 days after mixing.

\section{CONCLUSION}

AH Plus sealer had higher antibacterial effect than Ceraseal sealer. The incorporation of silver nanoparticles can improve the antibacterial activity of AH Plus and Ceraseal sealer at different time intervals. All the sealers showed the highest antibacterial efficacy when freshly mixed.

\section{REFERENCES}

1. Tabassum S, Khan FR. Failure of endodontic treatment: The usual suspects. Eur J Dent., 10:144, 2016.

2. Linsa RX Andradea AO, Juniorb RH, Wilsonc MJ, Michael AO ,David W. Williamsc DW, Fidel RAS. Antimicrobial resistance and virulence traits of Enterococcus faecalis from primary endodontic infections. Journal of Dentistry., 41:779-786, 2013

3. Haapasalo M, Endal U, Zandi H\& Coil JM. Eradication of endodontic infection by instrumentation and irrigation solutions. Endodontic Topics., 10: 77-102, 2005

4. Slenters TV, Hauser-Gerspach I, Daniels AU, and Fromm KM. Silver coordination compounds as light-stable, nanostructured and anti-bacterial coatings for dental implant and restorative materials. J Mat. Chem., 18:5359-5362, 2008.

5. Percival SL, Bowler PG, and Russel D. Bacterial resistance to silver in wound care. Journal of Hospital Infection., 60:1-7, 2005.

6. Schäfer, E.; Bering, N.; Bürklein, S. Selected physicochemical properties of AH Plus, EndoREZ and RealSeal SE root canal sealers. Odontology., 103: 61-65, 2015.
7. Neelakantan P, Sharma S, Shemesh H, Wesselink PR. Influence of Irrigation Sequence on the Adhesion of Root Canal Sealers to Dentin: A Fourier Transform Infrared Spectroscopy and Push-out Bond Strength Analysis. J. Endod., 41: 1108-1111, 2015.

8. Zhejun W. Bioceramic materials in endodontics. Endodontic Topics., 32: 3-30, 2015.

9. Ginebra MP, Fernandez E,Maeyer EAP. Setting reaction and hardening of an apatitic calcium phosphate cement. J Den Res., 76: 905-912, 1997.

10. Turkevich J, Stevenson P C, Hiller J. Discuss. Faraday Soc.,11: 55, 1951.

11. Lee P C, Meisel D. Adsorption and Surface Enhanced Raman of Dyes on Silver and Gold Sols. J Phy Chem., 86: 3391-3395, 1982.

12. Fabricius L, Dahlen G, Holm SE, Moller AJ. Influence of combinations of oral bacteria on periapical tissues of monkeys. Scand. J. Dent. Res., 90: 200-206, 1982.

13. Zhang H, Shen, N, Ruse D, and Haapasalo M. Antibacterial Activity of Endodontic Sealers by Modified Direct Contact Test Against Enterococcus faecalis. J Endod., 35:1051-1055, 2009

14. Stuart CH, Schwartz SA, Beeson TJ, Owatz CB. Enterococcus faecalis: its role in root canal treatment failure and current concepts in retreatment. J Endod., 32:93-98,2006.

15. Love RM. Enterococcus faecalis: a mechanism for its role in endodontic failure. Int Endod J., 34:399-405, 2001.

16. Haapasalo M, Qian W.Irrigants and intracanal medicaments. In: Ingle JI, Bakland LK, Baumgartner JC eds. Ingle's endodontics, 6th edn. Hamilton, ON, Canada: BC Decker Inc., 992-1011, 2008.

17. Ozbay G, Kitiki B, Peker S, Kargul B. Apical Sealing Ability of a Novel Material: Analysis by Fluid Filtration Technique. Acta Stomatol Croat., 48:132-9, 2014.

18. Nawal RR, Parande M, Sehgal R. A comparative evaluation of antimicrobial efficacy and flow properties for Epiphany, Guttaflow and AH- Plus sealer. Int Endod J., 44:307-13, 2011.

19. Nirupama DN, Nainan MT, Ramaswamy R. In vitro evaluation of the antimicrobial efficacy of four endodontic biomaterials against Enterococcus faecalis, Candida albicans and Styphylococcus aureus. Int J Biomater., 383756, 2014. 
20. Gholamhoseini Z, Alizadeh S, Bolbolian M. In vitro evaluation of antimicrobial activity of three bioceramic sealers against Enterococcus faecalis, and Styphylococcus aureus. Annals of Dental Specialty., 6, 2018.

21. Vibha H, Rathod R. Assessment of antimicrobial efficacy of bioceramic sealer, epiphany self-etch sealer, and $\mathrm{AH}-$ Plus sealer against Enterococcus faecalis, An in vitrostudy. Endodontology., 29:151-5, 2017.

22. Candeiro GT, Moura-Netto C, Almeida-Couto RSD, Azambuja-Junior N, Marques NMM, Cai S\&Gavini G. Cytotoxicity, genotoxicity and antibacterial effectiveness of a bioceramic endodontic sealer. Endod J., 49: 858-864, 2016.

23. Borges RP, Sousa-Neto MD, Versiani MA, Rached-Júnior FA, De- Deus G, Miranda CE. Changes in the surface of four calcium silicate- containing endodontic materials and an epoxy resin-based sealer after a solubility test. Int Endod J., 45:419-28, 2015.

24. Corrêa JM., Mori M., Sanches HL., Cruz AD, Poiate E \&Poiate IAVP. Silver nanoparticles in dental biomaterials. International Journal of Biomaterials., 1-9, 2015.

25. Masallat DT, Omar NS, Khalifa AK \&Emara RMK. Microbicidal Power of Silver Nano Particles and its Benefit in Soft Liner Obturator Prosthesis.EJMM., 25:4, 2016.

26. Cheng Z, Al Zaki A, Hui JZ. Multifunctional nanoparticles: cost versus benefit of adding targeting and imaging capabilities. Science., 338:903-10, 2012.

27. Krishnan R, Arumugam V, Vasaviah SK. The MIC and $\mathrm{MBC}$ of Silver Nanoparticles against Enterococcus faecalis - A Facultative Anaerobe. J NanomedNanotechnol., 6: $285,2015$.

28. Rai MK, Deshmukh SD, Ingle AP, Gade AK. Silver nanoparticles: the powerful nanoweapon against multi- drug-resistant bacteria. J Appl Microbiol., 112: 841-52, 2012.

29. Geetha RV, Veeraraghavan VP. Evaluation of antibacterial activity of five root canal sealants against Enterococcus faecalis - An in vitrostudy. Int J Pharm Sci Rev Res., 40:221-3, 2016.

30. Pizzo G, Giammanco GM, CumboE, Nicolosi G, Gallina G. In vitroantibacterial activity of endodontic sealers. J Dent., 34: 35-40, 2006.

31. Shakya VK. Antimicrobial Efficacy and Flow Characteristics of Four Root Canal Sealers an In-vitro Study. JCDR ., 10:104-108, 2016.

32. Cobankara FK, Altinöz HC, Ergani O, Kav K, Belli S. In vitroantibacterial activities of root-canal sealers by using two differentmethods. J Endod., 30:57-60, 2004.

33. Leonardo MR, Bezerra LA, Filho MT, Santana da Silva R. Release of formaldehyde by 4 endodontic sealers. Oral Surg. Oral Med. Oral Pathol. Oral Radiol. Endod., 88: 221$225,1999$.

34. Kayaoglu G, Erten H, Alacam T, Orstavik D. Short-term antibacterial activity of root canal sealers towards Enterococcus faecalis. Int. Endod. J., 38: 483-488, 2005.

35. Fuss Z, Charniaque O, Pilo R, Weiss E. Effect of various mixing ratios on antibacterial properties and hardness of endodontic sealers. J Endod., 26:519-22, 2000.

36. Gomes-Filho JE, Watanabe S, Lodi CS, Cintra LT, Nery MJ, Filho JA. Rat tissue reaction to MTA FILLAPEX. Dent Traumatol., 28:452-6, 2012.

37. Hasheminia M, Razavian H, Mosleh H, Shakerian B. In vitro evaluation of the antibacterial activity of five sealers used in root canal therapy. Dent Res J., 14:62-7, 2017. 